\title{
Localized measurement of longitudinal and transverse flow velocities in colloidal suspensions using optical coherence tomography
}

\author{
Nicolás Weiss, ${ }^{1, *}$ Ton G. van Leeuwen, ${ }^{1}$ and Jeroen Kalkman ${ }^{1,2}$ \\ ${ }^{1}$ Biomedical Engineering and Physics, Academic Medical Center, University of Amsterdam, \\ P.O. Box 22700, 1100 DE Amsterdam, the Netherlands \\ ${ }^{2}$ Quantitative Imaging Group, Faculty of Applied Sciences, Delft University of Technology, Lorentzweg 1, 2628 CJ Delft, the Netherlands
}

(Received 9 July 2013; published 28 October 2013)

\begin{abstract}
We report on localized measurement of the longitudinal and transverse flow velocities in a colloidal suspension using optical coherence tomography. We present a model for the path-length resolved autocorrelation function including diffusion and flow, which we experimentally verify. For flow that is not perpendicular to the incident beam, the longitudinal velocity gradient over the coherence gate causes additional decorrelation, which is described by our model. We demonstrate simultaneous imaging of sample morphology and longitudinal and transverse flow at micrometer scale in a single measurement.
\end{abstract}

DOI: 10.1103/PhysRevE.88.042312

PACS number(s): 83.85.Ei, 42.25.Kb, 83.50.Ha

\section{INTRODUCTION}

Modern experiments to study mass transport phenomena in complex rheological systems such as microfluidics [1], polymer solutions [2], biofilms [3], blood microcirculation [4], and blood [5,6] demand spatially and time resolved probing of concentration fields, pressure gradients, velocity profiles, wall shear stress, and diffusion coefficients. Optical techniques to measure fluid flow velocity are interesting because of their noncontact operation and high spatiotemporal resolution. Typically, optical techniques use a (coherent) light source to illuminate the sample and detect the fluctuations of the scattered light. Among conventionally used optical techniques are laser Doppler flowmetry (LDF) [7], laser speckle velocimetry (LSV) [8], and particle image velocimetry (PIV) [9]. In LDF, scattered light interferes with a local oscillator, and the intensity fluctuations of the detected light are related to the Doppler shift generated by flow in the sample. In LSV, the velocity of the scatterers is quantified by the intensity fluctuations that originate from the movement of the scatterers through the probing field. In PIV, the flow velocity is estimated by tracking the position over time of tracer particles present in the fluid. In LDF, LSV, and PIV the exact path-length distribution of the scattered light is unknown, making it impossible to quantify the velocity distribution deep inside the sample, thereby providing only volumetrically averaged information of the sample dynamics.

Optical coherence tomography (OCT) is an imaging technique in which low coherence interferometry is used to produce path-length resolved complex-valued backscatter profiles of (biological) samples up to a few millimeters deep [10]. In OCT, the transverse and longitudinal localization accuracies are given by the focusing optics and by the coherence of the light source, respectively. Functional extensions of OCT allow

\footnotetext{
*n.m.weiss@amc.uva.nl
}

Published by the American Physical Society under the terms of the Creative Commons Attribution 3.0 License. Further distribution of this work must maintain attribution to the author(s) and the published article's title, journal citation, and DOI. for localized measurements of static properties, such as layer thickness [11], birefringence [12], scattering and absorption coefficients [13], and scattering anisotropy [14], as well as sample dynamics, such as longitudinal flow [15] and particle diffusion $[16,17]$. Various studies have demonstrated that OCT can accurately determine the longitudinal component of the local flow velocity $[18,19]$. Determination of the transverse flow velocity has been pursued by analyzing the spectral bandwidth of the Doppler frequency shift [20-22], by using two distinct wave vectors for the incident light [23], by an autocorrelation method [24], by extracting the Doppler angle from the sample morphology [25], and by dynamic light scattering [26]. However, up until now no accurate quantification of the local transverse flow has been achieved.

In this paper, we present a theory for the path-length resolved OCT signal and its normalized autocorrelation function for the case of arbitrarily oriented flow in the presence of diffusion. We validate the theory by measuring the transverse and longitudinal flow velocities locally in a colloidal suspension. We show that sample morphology and flow velocity are determined simultaneously with high spatiotemporal resolution.

\section{MODEL FOR THE NORMALIZED AUTOCORRELATION OF THE OCT SIGNAL}

We start by writing the signal at the detector, following Ref. [27], as

$$
\begin{aligned}
i(t)= & \gamma \sqrt{P_{r}(t) P_{s}(t)} \operatorname{Re} \iiint_{-\infty}^{\infty} r\left(x-x_{s}, y-y_{s}, z-z_{s}\right) \\
& \times h(x, y) e^{-j 2 k(t) z} d x d y d z,
\end{aligned}
$$

where $\gamma$ is the photon-to-electron conversion efficiency, $P_{r}(t)$ is the reference arm power, $P_{s}(t)$ is the maximum sample arm power, $\mathrm{Re}$ is the real-part operator, $r(x, y, z)$ is the backscattering amplitude of the sample, $\left(x_{s}, y_{s}, z_{s}\right)$ is the position of the scattering sample, $h(x, y)$ is the profile of the transverse illuminating beam, and $k(t)=k_{0}+k_{1} t$ is the time dependent wave number, with $k=2 \pi / \lambda, t \in[-T / 2, T / 2]$, with $T$ being the period of the swept laser. We assume that 
the characteristic time constant of the scattering dynamics is sufficiently smaller than $T$.

The transverse illuminating beam is assumed to be Gaussian:

$$
h(x, y)=e^{-2 \frac{x^{2}}{w_{x}^{2}}} e^{-2 \frac{y^{2}}{w_{y}^{2}}},
$$

where $w_{x}$ and $w_{y}$ are the beam radii as the $(1 / \mathrm{e})$ radius of the field and the factor of 2 in the exponent accounts for the coupling efficiency of a single-mode fiber [28]. Note that the effect of the longitudinal confocal gate is neglected since for Fourier domain OCT systems the Rayleigh length is, in general, much larger than the coherence length (280 vs $8.1 \mu \mathrm{m}$ for the swept-source OCT system used here).

By inverse Fourier transforming Eq. (1) and assuming a Gaussian spectral envelope, the OCT signal $a_{O C T}(\hat{z}, t)$ is written as [27]

$$
\begin{aligned}
a_{O C T}(\hat{z}, t) \approx & \iiint_{-\infty}^{\infty} r\left(x-x_{s}, y-y_{s}, z-z_{s}\right) \\
& \times h(x, y) e^{-j 2 k_{0} z} e^{-\frac{[\hat{z}-z]^{2}}{w_{z}^{2}}} d x d y d z,
\end{aligned}
$$

where $w_{z}$ is the waist [(1/e) radius] of the coherence function.

For simplicity we restrict the analysis to the $(x, z)$ plane. Assuming that the reflection $r$ consists of $N$ point scatterers, Eq. (3) is written as

$$
\begin{aligned}
\operatorname{a}_{O C T}(\hat{z}, t) \approx & \int_{-\infty}^{\infty} \int_{\nu=1}^{N} \delta\left(x-x_{\nu}\right) \delta\left(z-z_{v}\right) \\
& \times h(x) e^{-j 2 k_{0} z} e^{-\frac{[\hat{z}-z]^{2}}{w_{z}^{2}}} d x d z,
\end{aligned}
$$

with $N$ being the total number of scatterers in the probed volume and $\left(x_{v}, x_{v}\right)$ being the position of the $v$ th scatterer. Now, solving the integrals, we write the OCT signal as

$$
a_{O C T}(\hat{z}, t) \approx \sum_{\nu=1}^{N} e^{-2 \frac{x_{\nu}^{2}}{w_{x}^{2}}} e^{-j 2 k_{0} z_{\nu}} e^{-\frac{\left[\hat{z}-z_{\nu}\right]^{2}}{w_{z}^{2}}} .
$$

Assuming stationarity, we write the normalized autocorrelation function as

$$
g(\hat{z}, \tau)=\left\langle a_{O C T}(\hat{z}, \tau) a_{O C T}^{*}(\hat{z}, 0)\right\rangle,
$$

where the asterisk indicates the complex-conjugate operation and the brackets indicate an ensemble average.

For the application described here we write the position of the scatterers as a summation of a diffusive and a directional component [29]:

$$
\begin{aligned}
& x_{v}(\tau)=x_{v}^{\prime}(\tau)+v_{x} \tau, \\
& z_{v}(\tau)=z_{v}^{\prime}(\tau)+v_{z} \tau .
\end{aligned}
$$

Assuming the diffusive motion to be independent of the initial position of the scatterers, we separate the ensemble average as the product of a diffusional and a translational average [29,30]:

$$
\begin{aligned}
g(\hat{z}, \tau)= & \sum_{\nu, \xi=1}^{N}\left\langle e^{-j 2 k_{0}\left[z_{v}^{\prime}(\tau)-z_{\xi}^{\prime}(0)\right]}\right\rangle\left\langle e^{-2 \frac{\left[x_{v}^{\prime}(\tau)+v_{x} \tau\right]^{2}}{w_{x}^{2}}} e^{-2 \frac{x_{\xi}^{\prime}(0)^{2}}{w_{x}^{2}}} e^{-j 2 k_{0} v_{z} \tau}\right. \\
& \left.e^{-\frac{\left\{\hat{z}-\left[z_{v}^{\prime}(\tau)+v_{z} \tau\right]\right\}^{2}}{w_{z}^{2}}} e^{-\frac{\left[\hat{z}-z_{\xi}^{\prime}(0)\right]^{2}}{w_{z}^{2}}}\right\rangle .
\end{aligned}
$$

Now, assuming that the scatterers are randomly placed throughout the scattering volume, all terms with $v \neq \xi$ in Eq. (8) are zero since the diffusive phase terms average out. Further, the sum can be dropped since all scatterers are assumed to be identical and all constant terms depending on $N$ are dropped. The diffusional average is known [31] and has been measured by OCT in Ref. [16]:

$$
\left\langle e^{-j 2 k_{0}\left[z^{\prime}(\tau)-z^{\prime}(0)\right]}\right\rangle=e^{-D q^{2} \tau}, \tau \geqslant 0,
$$

where $D$ is the diffusion coefficient given by the StokesEinstein equation $D=k_{B} T_{K} / 6 \pi \eta r$, with $k_{B}$ being Boltzmann's constant, $T_{K}$ being the absolute temperature, $\eta$ being the viscosity, and $r$ being the hydrodynamic particle radius. The absolute value of the scattering vector is $q=$ $4 \pi n \sin (\alpha / 2) / \lambda$, with $n$ being the refractive index of the medium, $\lambda$ being the wavelength in vacuum, and $\alpha$ being the scattering angle. Generally, OCT is performed in backscattering with low numerical aperture objectives. Further, we assume no number fluctuations, independence of particle concentration, and single scattering.

The translational average can be computed as the average over the initial position of the scatterers [30]:

$$
\begin{aligned}
& \left\langle e^{-2 \frac{\left[x_{v}^{\prime}(\tau)+v_{x} \tau\right]^{2}}{w_{x}^{2}}} e^{-2 \frac{x_{y}^{\prime}(0)^{2}}{w_{x}^{2}}} e^{-j 2 k_{0} v_{z} \tau} e^{-\frac{\left\{\hat{z}-\left[z_{v}^{\prime}(\tau)+v_{z} \tau\right]\right]^{2}}{w_{z}^{2}}} e^{-\frac{\left[\hat{z}-z_{v}^{\prime}(0)\right]^{2}}{w_{z}^{2}}}\right\rangle \\
& =\iint_{-\infty}^{\infty} e^{-2 \frac{\left[x+v_{x} \tau\right]^{2}}{w_{x}^{2}}} e^{-2 \frac{x^{2}}{w_{x}^{2}}} e^{-j 2 k_{0} v_{z} \tau} e^{-\frac{\left[\hat{z}-\left[z+v_{z} \tau\right]\right\}^{2}}{w_{z}^{2}}} e^{-\frac{[\hat{z}-z]^{2}}{w_{z}^{2}}} d x d z,
\end{aligned}
$$

where we have neglected the influence of diffusion on the amplitude terms. The transverse contribution is similar to that known in speckle velocimetry [8] and is calculated as

$$
\int_{-\infty}^{\infty} e^{-2 \frac{\left[x+v_{x} \tau\right]^{2}}{w_{x}^{2}}} e^{-2 \frac{x^{2}}{w_{x}^{2}}} d x=e^{-\frac{\left[v_{x} \tau\right]^{2}}{w_{x}^{2}}},
$$

where the spread of $v_{x}$ over the coherence gate is neglected.

Finally, the normalized autocorrelation function of the complex-valued OCT signal is written as

$$
\begin{aligned}
g(\hat{z}, \tau)= & e^{-D q^{2} \tau} e^{-\frac{\left[v_{x} \tau\right]^{2}}{w_{x}^{2}}} \int_{-\infty}^{\infty} e^{-j 2 k_{0} v_{z} \tau} \\
& \times e^{-\frac{\left[\hat{z}-\left.\left[z+v_{z} \tau\right]\right|^{2}\right.}{w_{z}^{2}}} e^{-\frac{[\hat{z}-z]^{2}}{w_{z}^{2}}} d z, \quad \tau \geqslant 0,
\end{aligned}
$$

where the exponential term describes the longitudinal diffusive dynamics, the Gaussian term in front of the integral describes the transverse directional dynamics, and the integral describes the longitudinal directional dynamics as a convolution of the Doppler phase term with the coherence detection gate. The normalization was taken with respect to $g(\hat{z}, 0)$. Note that this equation is similar to the one previously derived by Edwards et al. in the context of laser Doppler flowmetry [30]. The main difference here is that we have included the coherence detection gate in the derivation.

\section{MATERIALS AND METHODS}

\section{A. Optical coherence tomography system}

The experiments are performed with a home built fiberbased swept-source OCT system. The system operates at a 


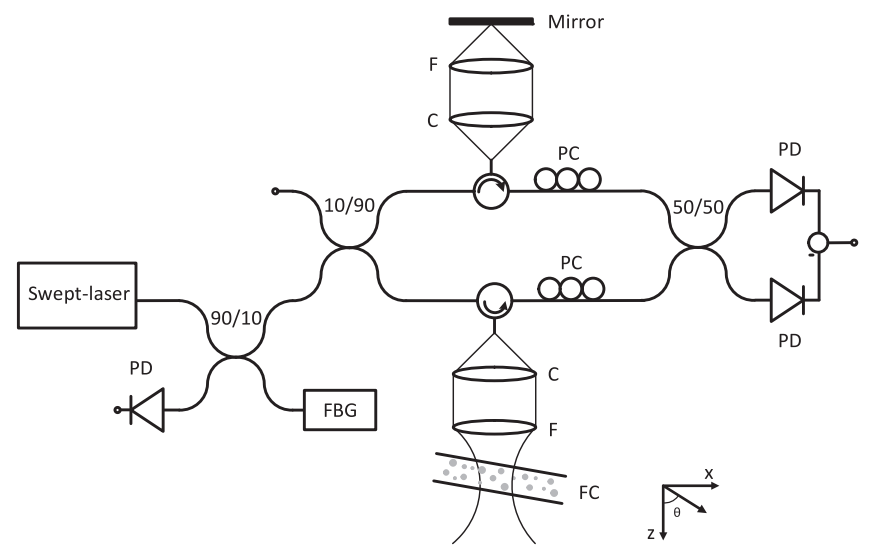

FIG. 1. Schematic of the experimental swept-source OCT setup. PD: photodetector, FBG: fiber Bragg grating, PC: polarization controllers, C: collimating lens, F: focusing lens, and FC: flow capillary. Gravity is in the $z$ direction.

center wavelength of $1312 \mathrm{~nm}$ with a bandwidth of $92 \mathrm{~nm}$ and a sweep frequency of $50 \mathrm{kHz}$ (Axsun Technologies). The average output power is $20.9 \mathrm{~mW}$, and the duty cycle is $59.4 \%$. Data are sampled (ATS9350, AlazarTech) with an interferometrically derived external clock signal at equidistant wave-number intervals. To ensure phase stability each sweep is triggered by the signal of a fiber Bragg grating centered at $1266 \mathrm{~nm}$ (OE Land) [32]. The interferometric signal is detected with a $150 \mathrm{MHz}$ balanced photodetector (PDB450C, Thorlabs) and a $80 \mathrm{MHz}$ low-pass filter (VLF-80+, Mini-Circuits). The trigger signal is detected with a $125 \mathrm{MHz}$ photodetector $(1811$, New Focus). The optics of the sample and reference arms are composed of a collimating lens (PAF-X-18-C, Thorlabs) and an achromatic doublet focusing lens (AC254-040-C, Thorlabs) with a numerical aperture of 0.04 . The power ratio of the sample and reference arms is $90: 10$. We measured $w_{x}=10.8 \pm 0.2 \mu \mathrm{m}$ and $w_{z}=8.1 \pm 0.3 \mu \mathrm{m}$ in air with a mirror reflector. For our OCT setup, the spread of $q$ over the bandwidth is small; therefore, we set $q=q_{c}$ at the center wavelength and $\alpha=180^{\circ}$ [16]. A schematic of the experimental setup is shown in Fig. 1.

Since the longitudinal decorrelation is determined by the coherence function of the light source, we measured it using a mirror reflector in air. The dots in Fig. 2 show the coherence gate of the swept light source measured by translating a mirror in the longitudinal direction mounted on a piezo-stage (M-664.164, Physik Instrumente) by steps of $1 \mu \mathrm{m}$. As can be seen, the coherence gate has asymmetric side lobes, which is attributed to the non-Gaussian shape of the light source. The coherence gate was fitted as the sum of two Gaussians, which are shown as the gray dashed lines in Fig. 2. The sum of the individual Gaussians is shown as the gray solid line. Note that the formalism presented in Sec. II is not restricted to a Gaussian spectrum of the light source, where the Fourier transform in Eq. (1) can be taken with an arbitrary spectral shape.

\section{B. Flow system}

Flow is generated by gravity and directed through a cylindrical glass capillary with an inner diameter of $562 \pm 25 \mu \mathrm{m}$. The

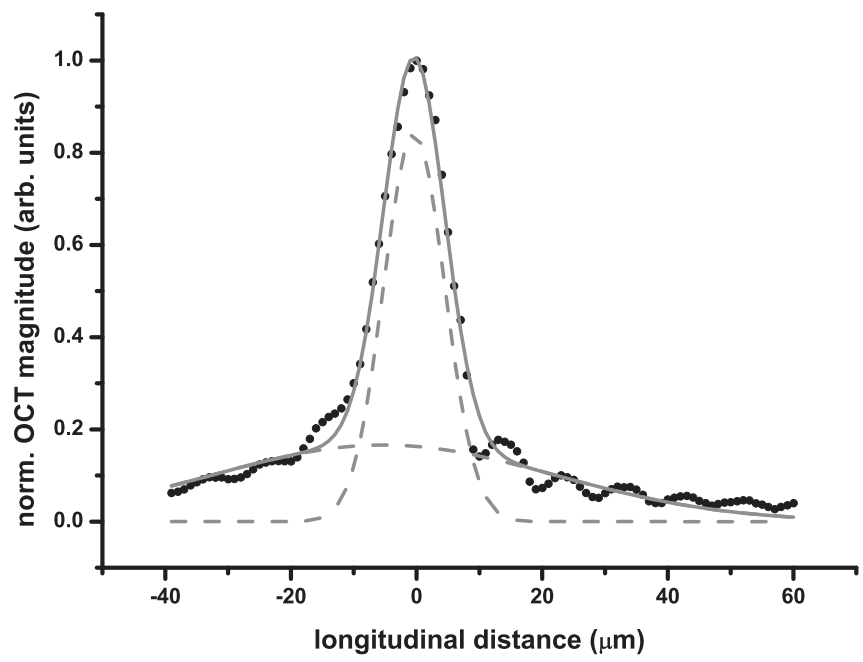

FIG. 2. Measured coherence gate fitted with the sum of two Gaussian functions. The dots represent the measured data, the gray solid line shows the fit to the data, and the gray dashed lines show the individual Gaussian functions.

flowing suspension consists of 1 vol \% Intralipid (Fresenius Kabi) dissolved in 74 vol \% distilled water and 25 vol \% glycerol (Acros Organics). The latter is used to reduce the Brownian motion of the particles in the suspension. The capillary is submerged in distilled water to reduce reflections from the outer glass walls. As a reference, the flow velocity is quantified by a mass discharge measurement. The measured refractive index of the medium is $n=1.38$.

\section{Data analysis}

Processing of the data is performed as follows: raw interferometric data consisting of 1088 data points are Fourier transformed to calculate the complex-valued OCT signal. For every path length we calculate the autocovariance of the real part of the OCT signal over 1000 time-adjacent acquisitions. This process is repeated 10 times and averaged. The longitudinal and transverse velocities are determined in the time domain by fitting the real part of Eq. (12) to the autocovariance of the data. In this fit, $v_{x}$ and $v_{z}$ are the free running parameters, while the diffusion coefficient of the colloidal suspension was set to $1.6 \pm 0.2 \mu \mathrm{m}^{2} \mathrm{~s}^{-1}$, as measured in a no-flow condition [16].

\section{RESULTS}

First, we analyze measurements for transverse flow, i.e., perpendicular to the optical beam $\left(\theta=90^{\circ}\right)$. Figures 3(a)-3(c) show log-log plots of the magnitude of the Fourier transform of Eq. (12) for three depths in the capillary overlaid on the experimental data. Figure 3(a) shows data for a depth close to the capillary wall $(z=-0.85 R)$. The gray dashed line shows the model in Eq. (12) with only the transverse directional term and without the contribution of diffusion. A clear discrepancy is observed for the higher frequency content of the signal. The gray solid line shows that including the effect of diffusion [the exponential term in Eq. (12)] demonstrates good agreement between the directional-diffusive model and 
(a)
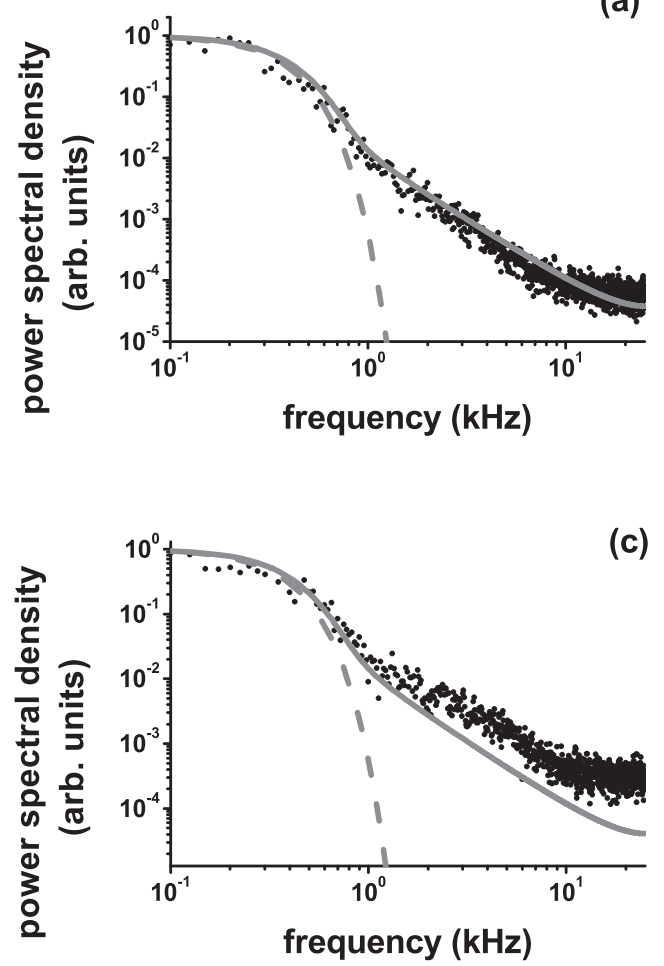

c)

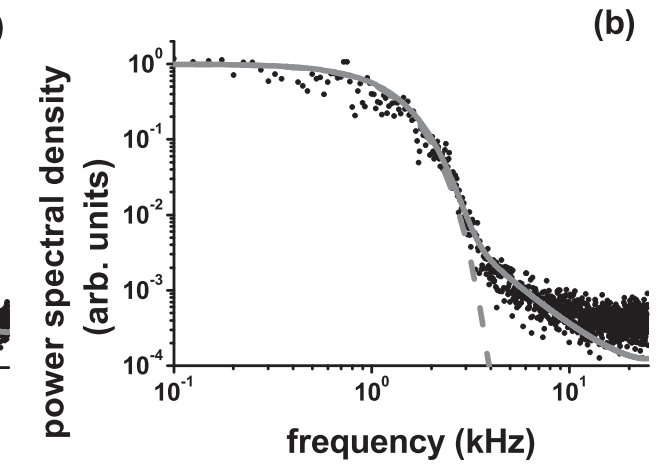

(b)

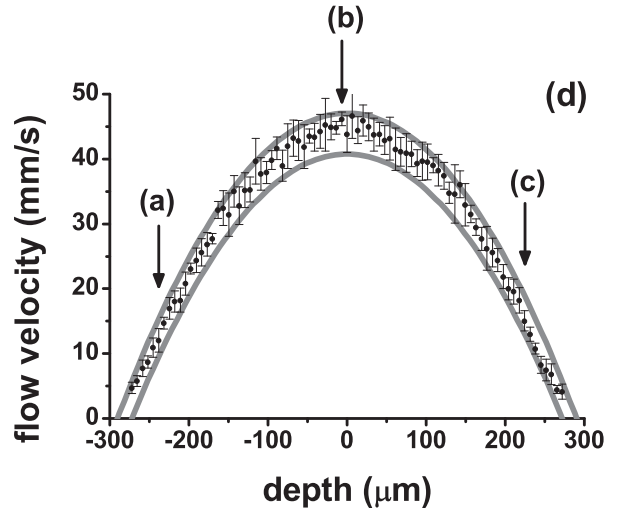

FIG. 3. Log-log plots of the power spectral density for a perpendicular measurement: (a) at a depth of $z=-0.85 R$ close to the capillary wall, (b) at the center, and (c) at a depth of $z=0.82 R$. The dots represent measured data, the gray dashed line represents the model with only directional dynamics, and the gray solid line represents the model including diffusion. (d) Depth resolved flow velocities in the capillary. The gray parabolas show the confidence interval of the reference velocity measurement. The arrows correspond to the depths shown in (a)-(c).

the data. Figure 3(b) shows a similar plot, but for a depth at the center of the capillary. The diffusive-directional model also agrees well with the data. The slight discrepancy at the larger frequencies is attributed to noise, which is not included in Eq. (12). Figure 3(c) shows data for a depth of $z=0.82 R$. As can be observed, the power at the higher frequencies is slightly larger $\left(10^{-4}\right.$ compared to $10^{-5}$ at $\left.20 \mathrm{kHz}\right)$ than predicted by theory. We attribute this to noise and multiple scattering present at larger depths, which can increase the linewidth of the power spectrum [19,33]. Figure 3(d) shows the measured depth resolved flow velocity profile through the capillary. The dots represent mean values over five measurements, and the error bars show the corresponding standard deviations. The gray parabolas show the confidence region of the reference velocity measurement. As can be observed, the computed flow velocity is in good agreement with the expected reference parabolas. The velocities obtained from the fit near the solid-liquid interface are slightly larger than expected from the reference. We attribute this to the relatively large effect of diffusion compared to the flow velocity at these locations on the OCT signal decorrelation.

Next, we analyze measurements for the nonperpendicular direction. Figure 4 shows results from an experiment in which the flow velocity vector is oriented at $\theta=79.5^{\circ}$. Again, Figs. 4(a)-4(c) show log-log plots of the magnitude of the Fourier transform of Eq. (12) overlaid on the measured data for three depths. Figure 4(a) shows data for a depth of $z=-0.89 R$ for two cases. For the first case (gray dashed line), the effect of the longitudinal velocity gradient over the coherence gate is neglected; that is, the integral in Eq. (12) is replaced by the Doppler phase term. As can be observed, the frequency at which the power spectrum peaks corresponds well to the Doppler frequency shift. However, the Gaussian and exponential terms describing the transverse directional and longitudinal diffusive dynamics are not sufficient to describe the broadening of the Doppler peak. For the second case (gray solid line), the integration of the coherence gate over the longitudinal flow profile is included, and as can be observed, the model is in good agreement with the measurement: the nonuniform distribution of Doppler frequencies in the scattering volume in the presence of a longitudinal velocity gradient explains the additional broadening of the Doppler peak [34]. Figure 4(b) shows a similar plot, but at a depth corresponding to the center of the capillary. Here, the additional broadening is negligible since at the center of the flow profile the flow gradient vanishes (gray dashed and solid lines are indistinguishable). Figure 4(c) shows data for the opposite side of the flow profile at a depth of $z=0.82 R$. The Doppler broadening is well described by including the gradient effect. Similar to the results shown in Fig. 3, at higher frequencies the measured power is larger than predicted by theory, which is attributed to the effect of multiple scattering at larger depths [19,33]. The power spectra shown in Figs. 4(a) and 4(c) are skewed in opposite directions. This is caused by the asymmetric shape of the coherence gate due to the non-Gaussian shape of the source's spectral shape (cf. Fig. 2). Figure 4(d) shows the measured depth resolved transverse and longitudinal flow velocities through the capillary using the reference method and a fit of 

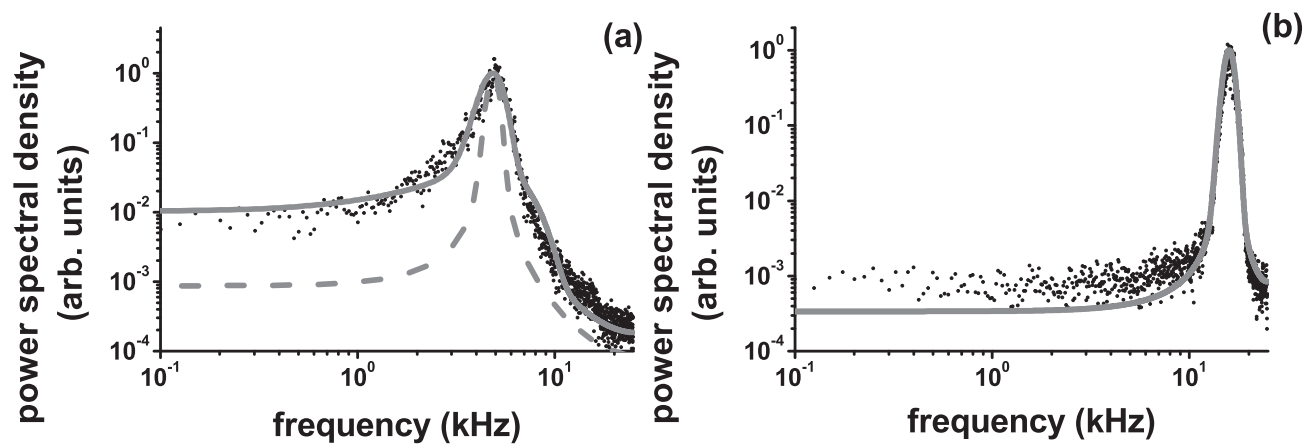

(b)

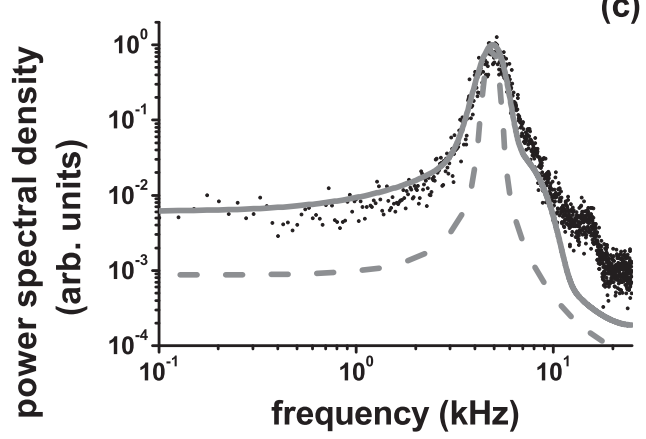

(c)

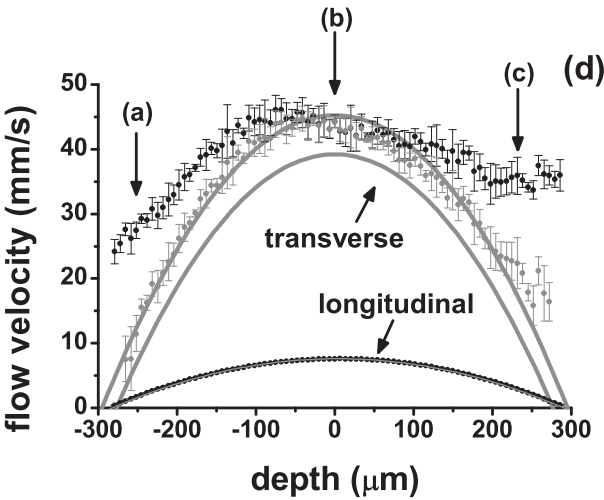

FIG. 4. Log-log plots of the power spectral density for a measurement on a tilted capillary at $\theta=79.5^{\circ}$ : (a) depth close to the wall of the capillary $z=-0.89 R$, (b) depth at the center, and (c) at a depth of $z=0.82 R$. The dots represent the measured data, the gray dashed line is the model without the convolution with the coherence gate, and the gray solid line includes it. (d) Depth resolved transverse and longitudinal flow velocities in the capillary for the two cases described above. The gray parabolas show the bounds of the reference velocity measurement. The arrows correspond to the depths shown in (a)-(c).

Eq. (12) to the data. The two higher flow velocity profiles correspond to the transverse velocity determined with and without the velocity gradient, and the lower flow velocity profile corresponds to the longitudinal velocity. The error bars for the longitudinal velocity are indistinguishable in the scale of the plot. The gray parabolas show the confidence intervals of the reference velocities. As can be observed, when ignoring the longitudinal velocity gradient within the measurement volume, the broadening of the Doppler peak shown in the previous figures translates into a significant overestimation of the transverse flow velocity (black dots) away from the center of the flow channel. The data that include the convolution of the coherence gate with the longitudinal phase term in Eq. (12) describe the expected transverse parabolic profile very well (gray dots). At large depths a small residual deviation of the transverse velocity is still observable, which we attribute to multiple scattering effects.

As an example application for simultaneous high speed imaging of depth resolved sample morphology and quantitative flow, Fig. 5 shows contour plots of the longitudinal and transverse flow velocities through the cross section of the capillary and the corresponding OCT magnitude image. The flow velocity vector is tilted at $\theta=87.4^{\circ}$. The images are composed of 37 depth resolved lines with a total acquisition time of 37 s. Using the described method, the measured maximum flow velocity $v_{\max }=\sqrt{v_{x, \max }^{2}+v_{z, \max }^{2}}=26.3 \pm 1.0 \mathrm{~mm} / \mathrm{s}$ at the center of the capillary is in good agreement with the reference velocity of $24.0 \pm 2.3 \mathrm{~mm} / \mathrm{s}$.

\section{DISCUSSION}

Our results show that we can determine with high accuracy both the longitudinal and transverse flow. The maximum longitudinal and transverse velocities that can be measured are determined by the Nyquist frequency of the time sampling, which is limited by the sweep rate of the laser [32]. The lower bound for the longitudinal velocity is determined by the signal-to-noise ratio [35]. The lower bound for the transverse velocity is determined by the fit of Eq. (12) and the dynamic time constants $\tau_{1}=\left(D q^{2}\right)^{-1}$ and $\tau_{2}=w_{x} / v_{x}$. For small transverse velocities the decorrelation of Eq. (12) is dominated by the diffusive term given that $\tau_{1} \ll \tau_{2}$. In the presence of diffusion an accurate estimation of small transverse velocities is therefore challenging. However, from our theoretical analysis we observe that a smaller value for $w_{x}$ increases the sensitivity towards the lower range of transverse velocities. However, a smaller $w_{x}$ decreases the sensitivity for the measurement of the diffusion coefficient [36]. The presented method can be used to determine with micrometer spatial resolution both the diffusion coefficient and the flow velocity from the OCT autocorrelation function simultaneously with the sample morphology. This provides an excellent testing ground for, e.g., the study of the behavior of the diffusion coefficient under shear stress, which is of paramount importance in studies of shear thinning [37], liquid-gel transitions [38], and anomalous diffusion [39]. Furthermore, using the presented technique, diffusion and flow near solid-liquid interfaces and in complex geometries can be 

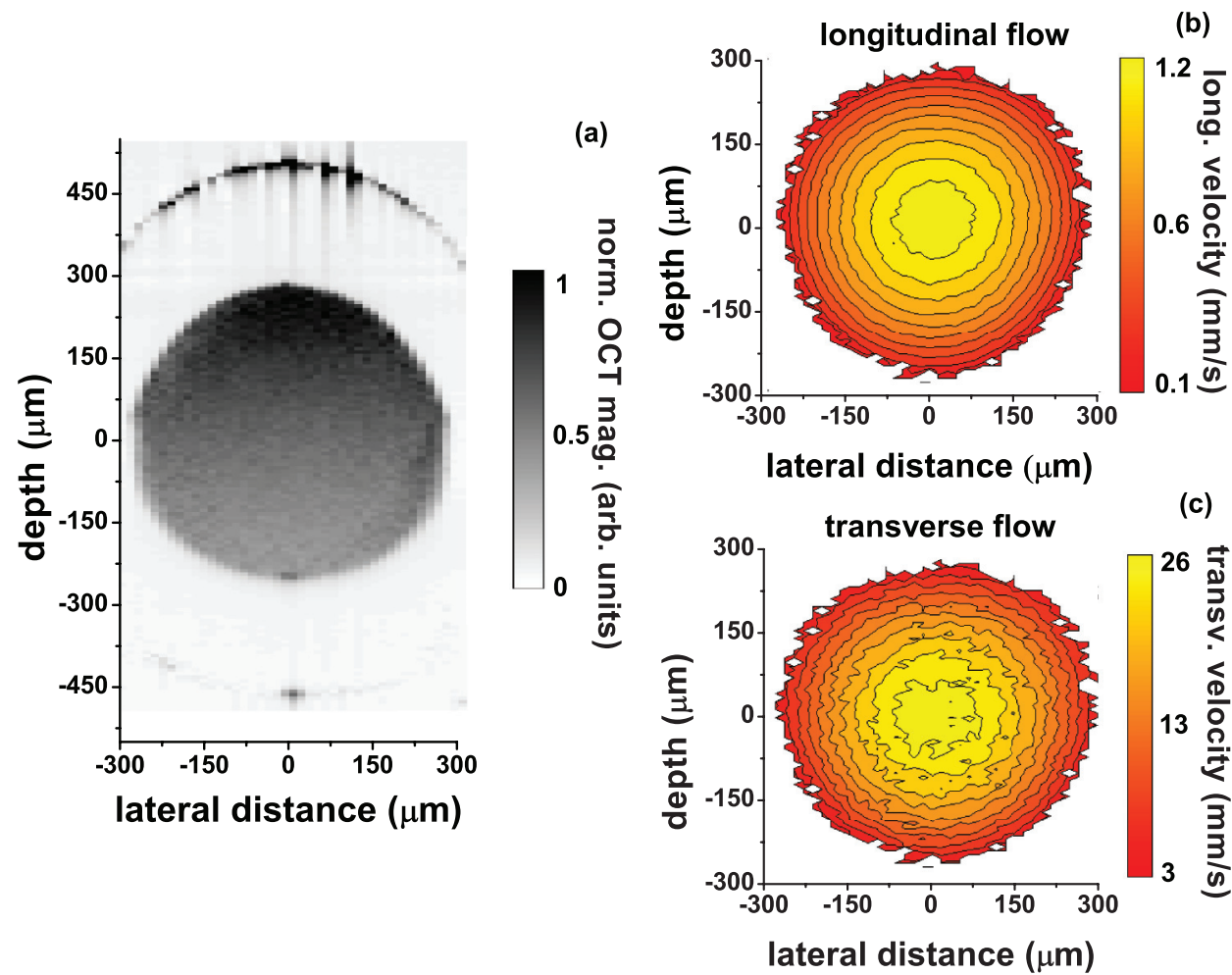

FIG. 5. (Color online) (a) OCT magnitude image of the tilted flow capillary. (b) and (c) Contour plots of the longitudinal and transverse flow velocities in the flow channel. The flow velocity is only computed in the region of interest determined by the OCT magnitude image.

studied. These effects are of particular importance in, e.g., hemodynamic research, where the shear rate has been shown to be a major factor in plaque rupture in arteries [40]. Finally, with the presented technique the effect of flow on rotational diffusion can be studied [17].

\section{CONCLUSION}

We have presented and validated a theory to quantify the local transverse and longitudinal directional dynamics of a colloidal suspension by measuring the path-length resolved autocorrelation function using optical coherence tomography. In contrast to the studies in Refs. [20-22,26], we have an analytical theoretical model, which incorporates the effects of the longitudinal flow velocity gradient and the diffusive dynamics to accurately measure the longitudinal and transverse flow velocity. Based on this model, we have obtained accurate results by fitting the model to the measured data with no free or unknown parameters. Our technique yields the local velocity and the sample morphology with high spatial and temporal resolution, which we demonstrated on flow in a capillary. We anticipate that the presented method opens up new opportunities for the study of a range of rheological properties of a variety of (non-)Newtonian fluids in complex flow geometries.

\section{ACKNOWLEDGMENTS}

We thank Anna Latour and Frank A. W. Coumans for discussions. This work was financially supported by the IOP Photonic Devices program.
[1] A. Günther and K. F. Jensen, Lab Chip 6, 1487 (2006).

[2] P. LeDuc, C. Haber, G. Bao, and D. Wirtz, Nature (London) 399, 564 (1999).

[3] P. Stoodley, D. de Beer, and Z. Lewandowski, Appl. Environ. Microbiol. 60, 2711 (1994).

[4] A. S. Popel and P. C. Johnson, Annu. Rev. Fluid Mech. 37, 43 (2005).

[5] X. Grandchamp, G. Coupier, A. Srivastav, C. Minetti, and T. Podgorski, Phys. Rev. Lett. 110, 108101 (2013).

[6] M. B. Lawrence and T. A. Springer, Cell 65, 859 (1991).

[7] Y. Yeh and H. Z. Cummins, Appl. Phys. Lett. 4, 176 (1964).

[8] J. Ohtsubo and T. Asakura, Opt. Quantum Electron. 8, 523 (1976).
[9] R. J. Adrian, Annu. Rev. Fluid Mech. 23, 261 (1991)

[10] D. Huang, E. Swanson, C. P. Lin, J. S. Schuman, W. G. Stinson, W. Chang, M. R. Hee, T. Flotte, K. Gregory, C. A. Puliafito, and J. G. Fujimoto, Science 254, 1178 (1991).

[11] W. Drexler, U. Morgner, R. K. Ghanta, F. X. Kärtner, J. S. Schuman, and J. G. Fujimoto, Nat. Med. 7, 502 (2001).

[12] J. F. de Boer, T. E. Miller, M. J. C. van Gemert, and J. S. Nelson, Opt. Lett. 22, 934 (1997).

[13] D. J. Faber, M. C. G. Aalders, E. G. Mik, B. A. Hooper, M. J. C. van Gemert, and T. G. van Leeuwen, Phys. Rev. Lett. 93, 028102 (2004).

[14] V. M. Kodach, D. J. Faber, J. van Marle, T. G. van Leeuwen, and J. Kalkman, Opt. Express 19, 6131 (2011). 
[15] Z. Chen, T. E. Milner, D. Dave, and J. S. Nelson, Opt. Express 22, 64 (1997).

[16] J. Kalkman, R. Sprik, and T. G. van Leeuwen, Phys. Rev. Lett. 105, 198302 (2010).

[17] R. K. Chhetri, K. A. Kozek, A. C. Johnston-Peck, J. B. Tracy, and A. L. Oldenburg, Phys. Rev. E 83, 040903(R) (2011).

[18] M. D. Kulkarni, T. G. van Leeuwen, S. Yazdanfar, and J. A. Izatt, Opt. Lett. 23, 1057 (1998).

[19] J. Kalkman, A. V. Bykov, D. J. Faber, and T. G. van Leeuwen, Opt. Express 18, 3883 (2010).

[20] H. Ren, K. M. Brecke, Z. Ding, Y. Zhao, J. Stuart Nelson, and Z. Chen, Opt. Lett. 27, 409 (2002).

[21] D. Piao, L. L. Otis, and Q. Zhu, Opt. Lett. 28, 1120 (2003).

[22] S. G. Proskurin, Y. He, and R. K. Wang, Opt. Lett. 28, 1227 (2003).

[23] D. P. Davé and T. E. Milner, Opt. Lett. 25, 1523 (2000).

[24] Y. Wang and R. Wang, Opt. Lett. 35, 3538 (2010).

[25] R. Michaely, A. H. Bachmann, M. L. Villiger, C. Blatter, T. Lasser, and R. A. Leitgeb, J. Biomed. Opt. 12, 041213 (2007).

[26] J. Lee, W. Wu, J. Y. Jiang, B. Zhu, and D. A. Boas, Opt. Express 20, 22262 (2012).

[27] S. H. Yun, G. J. Tearney, J. F. de Boer, and B. E. Bouma, Opt. Express 12, 2977 (2004).
[28] M. Gu, C. J. R. Sheppard, and X. Gan, J. Opt. Soc. Am. A 8, 1755 (1991).

[29] D. P. Chowdhury, C. M. Sorensen, T. W. Taylor, J. F. Merklin, and T. W. Leste, Appl. Opt. 23, 4149 (1984).

[30] R. V. Edwards, J. C. Angus, M. J. French, and J. W. Dunning, Jr., J. Appl. Phys. 42, 837 (1971).

[31] C. S. Johnson, Jr., and D. A. Gabriel, Laser Light Scattering (Dover, New York, 1981).

[32] H. C. Hendargo, R. P. McNabb, A. Dhalla, N. Shepherd, and J. A. Izatt, Biomed. Opt. Express 2, 2175 (2011).

[33] K. K. Bizheva, A. M. Siegel, and D. A. Boas, Phys. Rev. E 58, 7664 (1998).

[34] To evaluate Eq. (12) the necessary analytical expression for the longitudinal flow velocity is derived from the Doppler shift measurement.

[35] M. A. Choma, A. K. Ellerbee, S. Yazdanfar, and J. A. Izatt, J. Biomed. Opt. 11, 024014 (2006).

[36] T. W. Taylor and C. M. Sorensen, Appl. Opt. 25, 2421 (1986).

[37] Y. Xia and P. T. Callaghan, Macromolecules 24, 4777 (1991).

[38] G. Popescu, A. Dogariu, and R. Rajagopalan, Phys. Rev. E 65, 041504 (2002).

[39] H. Orihara and Y. Takikawa, Phys. Rev. E 84, 061120 (2011).

[40] S. D. Gertz and W. C. Roberts, Am. J. Cardiol. 66, 1368 (1990). 